\title{
Influence des lois de mouvement sur les déformations et les vibrations des machines à grande vitesse
}

\author{
DANiEL BRUn-PiCARD ${ }^{\mathrm{a}}$ \\ LSIS - UMR CNRS 6168, Équipe IMS, ENSAM, 2 cours des Arts et Métier, 13617 Aix-en-Provence Cedex 1, France
}

Reçu le 14 février 2005, accepté le 13 avril 2005

\begin{abstract}
Résumé - Lorsqu'on cherche à réduire les écarts de suivi de trajectoire, la structure de la machine est astreinte à des accélérations susceptibles d'engendrer des déformations et des vibrations d'autant plus intenses que la vitesse exigée est élevée. Le choix de la trajectoire et de la loi de mouvement n'est pas anodin car, selon les cas, on peut observer des différences substantielles d'amplitude de déformation et de vibration. Il s'agit alors de déterminer des trajectoires et des lois de mouvement réalistes, que les asservissements soient capables de suivre sans erreur de poursuite ni vibration induite. Différentes voies de génération de lois de mouvement optimisées sont présentées, avec une analyse du gain qu'elles peuvent apporter.
\end{abstract}

Mots clés : Écart de poursuite / vibration / loi de mouvement / optimisation

Abstract - Influence of motion laws on the deformations and the vibrations of high-speed machines. When we try to reduce the tracking errors, the machines are compelled to high accelerations, liable to generate all the more intense deformations and vibrations as the required speed is higher. The choice of the motion laws is not insignificant because, according to cases, we can observe substantial differences in amplitude of deformations and vibrations. Various ways of generation of optimised motion laws and feed-forward control are presented in this article, with an analysis of their advantages.

Key words: Tracking errors / vibration / motion law / optimisation

\section{Introduction}

Les exigences croissantes de productivité et de qualité imposent la mise en œuvre de la grande vitesse en même temps que l'amélioration de la précision, de la répétabilité et de la fiabilité des machines. Cette évolution a suscité une remise en cause totale non seulement des architectures des machines et des choix de technologie (matériaux, structures, construction, actionnement, instrumentation, commande), mais aussi de la façon de concevoir et d'utiliser ces machines. La performance ne peut en effet être obtenue qu'au prix d'une prise en compte de l'ensemble des savoirs et savoir-faire des spécialistes de ces technologies dès le début et tout au long de la conception des machines. Le problème se situe donc, actuellement et conjointement aux niveaux :

- de la conception de la machine dont les flexibilités inévitables doivent affecter le moins possible les opérations que l'on veut lui faire réaliser;

a Auteur pour correspondance :

daniel.brun-picard@aix.ensam.fr
- de la commande de la machine qui doit réduire les écarts de poursuite et les vibrations par l'application de techniques de contrôle actif;

- de la définition de trajectoires et loi de mouvement permettant d'éviter ou de corriger les écarts et les vibrations, ce qui, dans le cas d'opérations d'usinage, impacte la conception des pièces, certaines formes étant irréalisables à grande vitesse.

Sur les aspects relevant de la conception des machines, on peut se référer à la publication d'Antoine Dequidt et al. [1] qui propose une approche intégrant la commande et la conception des chaînes d'actionnement et à la thèse de François Debrouke [2], qui donne des démarches et préconisations de conception des machines pour éliminer ou réduire leur basculement sous accélération.

Sur les aspects « commande», les travaux ont tout d'abord porté sur l'amélioration des performances des boucles d'asservissement en termes de précision, rapidité et robustesse [3], puis sur des techniques de contrôle actif pour limiter le niveau des vibrations [4]. La précision de suivi de trajectoire à grande vitesse implique l'application de gains de contre-réaction très élevés et, donc, 
l'accroissement de la bande passante. Les déformations et les vibrations des constituants de la machine ne sont ainsi plus seulement sollicitées par les efforts de coupe (en usinage), mais aussi, et de façon de plus en plus prépondérante, par l'intensité et la rapidité de variations des forces et/ou couples développés par les actionneurs. Ces phénomènes sont renforcés par l'application de techniques d'anticipation visant à annuler l'écart de poursuite des asservissements.

En ce qui concerne la génération de trajectoires et de lois de mouvement, on peut observer que plus l'écart de poursuite est faible, plus les organes de la machine sont astreints à suivre le mouvement demandé. Dans le passé, la commande bénéficiait d'une certaine « mollesse » et lissait les consignes lorsqu'elles étaient irréalistes. Dorénavant, si on ne veut pas d'erreur ni de vibration, il faut impérativement appliquer des consignes qui respectent les possibilités de la machine, compte tenu des inerties et des flexibilités de ses organes. Les phénomènes physiques rencontrés étant incontournables, le but n'est donc plus seulement de tenter de contraindre le système mécanique à suivre une trajectoire définie à partir uniquement de considérations géométriques, mais d'accommoder les trajectoires et les vitesses désirées aux lois de la mécanique. Sur ce point, il n'y a actuellement que peu de travaux publiés, mis à part ceux de Serge Bloch $[5,6]$ et de Richard Béarée [7]. Serge Bloch, qui s'intéressait aux trajectoires d'usinage, préconisait d'adapter la vitesse en fonction de la forme de la trajectoire, en ralentissant pour les courbes. Richard Béarée, qui s'intéresse au positionnement, préconise un contrôle du jerk. Il démontre l'efficacité de ce contrôle dans un cas spécifique, à la fois de façon analytique et de façon expérimentale.

Le présent article se place dans une optique plus globale et tente par la voie théorique de déterminer quelles sont les lois de mouvement qui minimisent les vibrations indésirables. Les développements présentés ici ont ainsi un caractère plutôt mathématique, mais débouchent sur des préconisations très proches de la pratique.

\section{Lois de mouvement optimales en positionnement point à point rapide}

Le positionnement point à point se caractérise par le fait que l'on veut atteindre une position déterminée dans un délai fixé et s'arrêter sur cette position. Le mouvement est libre entre la position initiale et la position finale.

La présente étude est limitée aux cas de machines cartésiennes, pour lesquelles les mouvements ne sont pas couplés et peuvent s'exécuter indépendamment et simultanément. On peut donc considérer le mouvement de chaque axe séparément.

\section{1 Équation du mouvement de l'axe à commander, incluant un mode vibratoire}

Le système considéré est l'ensemble de l'axe, avec son asservissement en boucle fermée. Son comportement peut être représenté par une équation différentielle d'ordre $n$ ( $n \geq 2$ pour inclure au moins un mode vibratoire). Soit :

$a_{n} y^{(n)}(t)+a_{n-1} y^{(n-1)}(t)+\ldots+a_{1} y^{\prime}(t)+y(t)=u(t)+p(t)$

$y^{(n)}(t)$ : dérivée $n$-ième de la grandeur asservie $y(t)$;

$u(t)$ : fonction de commande dépendant de la loi de mouvement désirée;

$p(t)$ : fonction traduisant les perturbations ;

$a_{1}, a_{2} \ldots a_{n}$ : coefficients du dénominateur de la fonction de transfert en boucle fermée.

Idéalement, la fonction de commande $u(t)$ doit être de la forme :

$$
u(t)=a_{n} y_{\mathrm{d}}^{(n)}(t)+a_{n-1} y_{\mathrm{d}}^{(n-1)}(t)+\ldots+a_{1} y_{\mathrm{d}}^{\prime}(t)+y_{\mathrm{d}}(t)
$$

$y_{\mathrm{d}}(t)$ : loi de mouvement désirée.

Ceci garantit un écart de poursuite $\varepsilon(t)=y_{\mathrm{d}}(t)-y(t)$ tel que :

$$
a_{n} \varepsilon^{(n)}(t)+a_{n-1} \varepsilon^{(n-1)}(t)+\ldots+a_{1} \varepsilon^{\prime}(t)+\varepsilon(t)=p(t)
$$

Sous cette forme, on voit que l'écart de poursuite ne dépend que de l'erreur initiale et des perturbations. L'asservissement étant a priori réglé de façon à ce que le système soit stable et rapide, l'effet de l'erreur initiale n'apparaît qu'à la mise en marche de l'asservissement et s'estompe en très peu de temps, avec la rapidité propre à la boucle d'asservissement.

La forme de l'équation (2) démontre que, pour que la commande soit réalisable il faut que la consigne et ses dérivées jusqu'à l'ordre $n$ compris ne soient pas trop grande, ce qui exclut toute discontinuité de la consigne et de ses dérivées jusqu'à l'ordre $n-1$.

La mise en œuvre de la commande définie par l'équation (2) impose que la consigne et ses dérivées soient connues à chaque instant, ce qui n'est pas un problème du fait que cette consigne est délivrée par un « générateur de trajectoire » qui élabore une loi de mouvement par interpolation, en utilisant des fonctions continues et dérivables.

Une difficulté réelle existe dans le cas des systèmes à déphasage non minimal. Leur fonction de transfert en boucle ouverte présente un ou plusieurs zéros à partie réelle positive qu'il est impossible d'éliminer par les boucles de contre-réaction. Ces zéros demeurent donc présents dans la fonction de transfert en boucle fermée. Pour prendre en compte le numérateur de la fonction de transfert du système en boucle fermée, il faut écrire la relation entre $u(t)$ et l'entrée de consigne du système bouclé $y_{\mathrm{c}}(t)$ :

$$
u(t)=b_{n} y_{\mathrm{c}}^{(n)}(t)+b_{n-1} y_{\mathrm{c}}^{(n-1)}(t)+\ldots+b_{1} y_{\mathrm{c}}^{\prime}(t)+b_{0} y_{\mathrm{c}}(t)
$$

$b_{1}, b_{2} \ldots b_{n}$ : coefficients du numérateur de la fonction de transfert en boucle fermée.

Le calcul de la loi de consigne à partir de la loi de commande implique la résolution de l'équation différentielle (4). Ceci ne pose pas de problème s'il n'y 
a pas de zéros à partie réelle positive. Par contre, s'il y en a, l'équation (4) est instable. Si la dynamique de l'instabilité est lente devant le temps d'exécution du mouvement point à point cette instabilité n'est pas gênante car la consigne $y_{\mathrm{c}}(t)$ n'a pas le temps d'atteindre des valeurs élevées. Sinon, la seule solution restante est d'imposer à la loi de mouvement désirée $y_{\mathrm{d}}(t)$ de ne pas exciter l'instabilité, ce qui implique que la transformée de Laplace de cette loi de mouvement doit inclure les mêmes zéros à partie réelle positive que le système commandé. Ce cas ne sera pas étudié plus en détail ici car il pourrait faire l'objet à lui seul d'un papier complet.

\subsection{Détermination analytique d'une loi de mouvement n'excitant pas les vibrations}

Il existe a priori une infinité de lois de mouvement permettant d'aller d'un point à un autre sans engendrer de vibration. La question est alors de chercher qu'elle est meilleure par rapport à un critère que l'on doit définir. Il est connu que les grandeurs pénalisantes pour la réalisation d'un mouvement sont la vitesse qui cause des pertes par frottement et par courant de Foucault, l'accélération, qui cause des pertes par effet Joule ${ }^{1}$ et le jerk qui cause des variations de contraintes et des vibrations nuisibles. Le critère ainsi proposé est :

$$
J\left(y_{\mathrm{d}}(t)\right)=\frac{1}{2} \int_{0}^{T}\left(\lambda_{\mathrm{j}} \dddot{y}_{\mathrm{d}}^{2}(t)+\lambda_{\mathrm{a}} \ddot{y}_{\mathrm{d}}^{2}(t)+\lambda_{\mathrm{v}} \dot{y}_{\mathrm{d}}^{2}(t)\right) \mathrm{d} t
$$

Le mouvement est supposé devoir s'exécuter pendant l'intervalle de temps $0 \leq t \leq T . \lambda_{\mathrm{j}}, \lambda_{\mathrm{a}}$ et $\lambda_{\mathrm{v}}$, sont des coefficient de pénalisation du jerk, de l'accélération et de la vitesse.

Il s'agira alors de déterminer la loi de mouvement $\left\{y_{\mathrm{d}}(t), 0 \leq t \leq T\right\}$, telle que le résultat de l'intégrale soit le plus petit possible. Les termes sous l'intégrale peuvent être interprétés comme des puissances dissipées à chaque instant par l'excitation des vibrations, par l'effet Joule et par les frottements et courants de Foucault. L'intégrale est assimilable à une énergie dépensée pour effectuer le mouvement. On reste cependant libre de s'écarter de l'énergie dissipée au sens physique et de moduler le poids de chaque terme en adaptant les coefficients de pénalisation. Par exemple, pour privilégier la réduction des vibrations, on peut choisir un coefficient $\lambda_{\mathrm{j}}$ très grand.

Pour résoudre ce problème d'optimisation, il faut considérer une petite variation de la loi de mouvement telle que $y_{\mathrm{d}}(t) \rightarrow y_{\mathrm{d}}(t)+\partial y_{\mathrm{d}}(t)$. Au voisinage du minimum du critère, la variation de celui-ci doit être nulle au premier ordre et positive au second ordre. Dès lors que les coefficients $\lambda$ sont positifs, on est assuré de la positivité de la variation du second ordre grâce à la forme de type

\footnotetext{
${ }^{1}$ Dans les actionneurs rapides, le terme prépondérant du
} courant de commande est proportionnel à l'accélération. quadratique du critère. Pour ce qui est du premier ordre, en différentiant l'équation (5), il vient :

$$
\begin{aligned}
\partial J=\int_{0}^{T}\left(\lambda_{\mathrm{j}} \dddot{y}_{\mathrm{d}}(t) \partial \dddot{y}_{\mathrm{d}}(t)\right. \\
\left.\quad+\lambda_{\mathrm{a}} \ddot{y}_{\mathrm{d}}(t) \partial \ddot{y}_{\mathrm{d}}(t)+\lambda_{\mathrm{v}} \dot{y}_{\mathrm{d}}(t) \partial \dot{y}_{\mathrm{d}}(t)\right) \mathrm{d} t
\end{aligned}
$$

Pour se ramener à $\partial y_{\mathrm{d}}(t)$, il y a lieu d'intégrer l'équation (6) par parties à trois reprises, à cause du terme $\partial \dddot{y}_{\mathrm{d}}(t)$. Cela conduit à :

$$
\begin{aligned}
\partial J & =\left(\lambda_{\mathrm{j}} \dddot{y}_{\mathrm{d}}(t) \partial \ddot{y}_{\mathrm{d}}(t)+\lambda_{\mathrm{a}} \ddot{y}_{\mathrm{d}}(t) \partial \dot{y}_{\mathrm{d}}(t)\right. \\
& \left.+\lambda_{\mathrm{v}} \dot{y}_{\mathrm{d}}(t) \partial y_{\mathrm{d}}(t)\right)\left.\right|_{0} ^{T} \\
& -\left.\left(\lambda_{\mathrm{j}} \ddot{y}_{\mathrm{d}}(t) \partial \dot{y}_{\mathrm{d}}(t)+\lambda_{\mathrm{a}} \dddot{y}_{\mathrm{d}}(t) \partial y_{\mathrm{d}}(t)\right)\right|_{0} ^{T} \\
& +\left.\left(\lambda_{\mathrm{j}} \ddot{\ddot{y}}_{\mathrm{d}}(t) \partial y_{\mathrm{d}}(t)\right)\right|_{0} ^{T} \\
& -\int_{0}^{T}\left(\lambda_{\mathrm{j}} \ddot{y}_{\mathrm{d}}(t)-\lambda_{\mathrm{a}} \ddot{y}_{\mathrm{d}}(t)+\lambda_{\mathrm{v}} \ddot{y}_{\mathrm{d}}(t)\right) \partial y_{\mathrm{d}}(t) \mathrm{d} t=0
\end{aligned}
$$

La notation « $\left.\lambda y_{\mathrm{d}}^{(n)}(t) \partial y_{\mathrm{d}}^{(m)}(t)\right|_{0} ^{T} »$, utilisée pour représenter les termes qui sont sortis hors de l'intégrale, signifie qu'il faut faire la soustraction entre la valeur finale et la valeur initiale. On a donc $\left.\lambda y_{\mathrm{d}}^{(n)}(t) \partial y_{\mathrm{d}}^{(m)}(t)\right|_{0} ^{T}=$ $\lambda y_{\mathrm{d}}^{(n)}(T) \partial y_{\mathrm{d}}^{(m)}(T)-\lambda y_{\mathrm{d}}^{(n)}(0) \partial y_{\mathrm{d}}^{(m)}(0)$. À l'instant initial et à l'instant final, les positions, vitesses et accélérations sont imposées par les conditions aux limites que l'on veut respecter. On ne peut donc pas les faire varier, ce qui implique que tous les $\partial y_{\mathrm{d}}^{(m)}(0)$ et $\partial y_{\mathrm{d}}^{(m)}(T)$ sont nuls. L'équation (7) se résume donc à :

$$
\partial J=-\int_{0}^{T}\left(\lambda_{j} \ddot{y}_{\mathrm{d}}(t)-\lambda_{a} \ddot{\ddot{y}}_{\mathrm{d}}(t)+\lambda_{v} \ddot{y}_{\mathrm{d}}(t)\right) \partial y_{\mathrm{d}}(t) \mathrm{d} t=0
$$

Pour que $\partial J$ soit nul quelles que soient les variations $\partial y_{\mathrm{d}}(t)$ à tous les instants autres qu'initiaux et finaux, il faut et il suffit que l'expression entre parenthèses, dans l'intégrale, soit nulle, ce qui donne :

$$
\lambda_{\mathrm{j}} \dddot{\dddot{y}}_{\mathrm{d}}(t)-\lambda_{\mathrm{a}} \ddot{y}_{\mathrm{d}}(t)+\lambda_{\mathrm{v}} \ddot{y}_{\mathrm{d}}(t)=0
$$

Il s'agit d'une équation différentielle linéaire, dont la résolution ne pose pas de problème particulier et conduit à une loi de mouvement associant des fonctions exponentielles et sinusoïdales. La figure 1 donne les profils de position, vitesse, accélérations obtenus pour réaliser un déplacement point à point unitaire en une unité de temps, avec $\lambda_{\mathrm{j}}=1, \lambda_{\mathrm{a}}=0, \lambda_{\mathrm{v}}=10^{6}$. Le fait que $\lambda_{\mathrm{v}}$ soit très grand signifie que l'on souhaite minimiser de façon prépondérante la vitesse. Ceci permet d'obtenir une vitesse quasi constante, sauf pendant les phases de démarrage et d'arrêt.

$\mathrm{Du}$ fait que l'on souhaite atteindre rapidement la vitesse de croisière, le jerk prend des valeurs initiales et finales relativement élevées. On pourrait s'attendre à ce que cela produise une forte excitation des vibrations. Mais, comme les conditions initiales définissent des points 

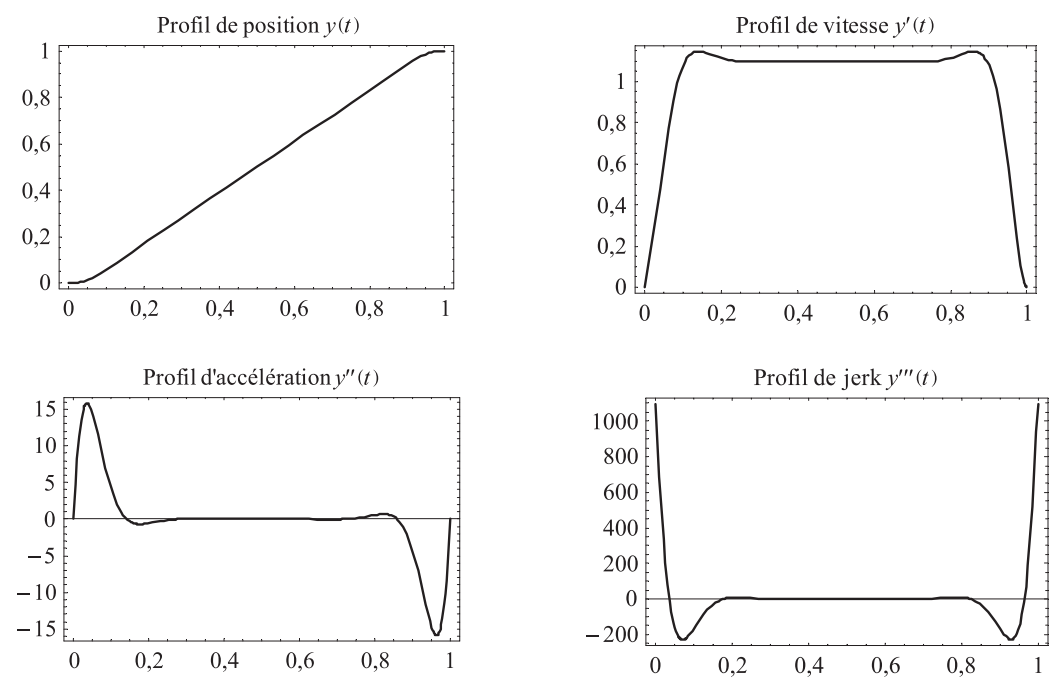

Fig. 1. Loi de mouvement point à point optimale pour $\lambda_{\mathrm{j}}=1, \lambda_{\mathrm{a}}=0, \lambda_{\mathrm{v}}=10^{6}$.
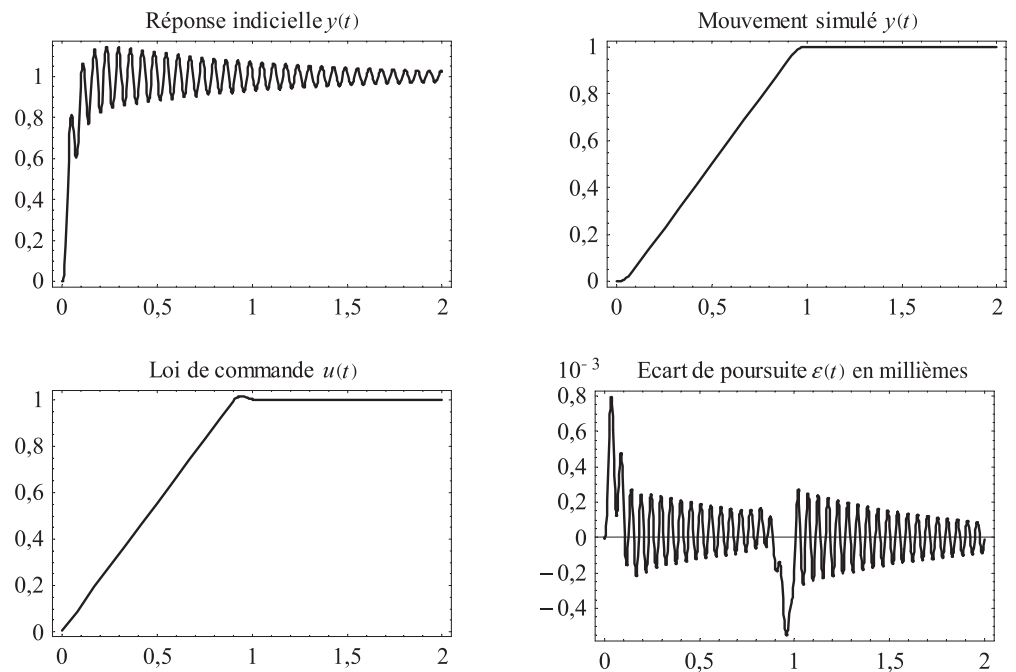

Fig. 2. Comportement du système simulé ${ }^{2}$.

d'équilibre (vitesse et accélération nulles) et comme, aussi, la loi de commande donnée par l'équation (2) impose au système de suivre une loi de mouvement exempte d'oscillation, il n'y a pas de vibration ni pendant le mouvement, ni à la fin de celui-ci.

Le jerk étant discontinu aux instants initiaux et finaux, cette loi de mouvement ne sera pas réalisable par un système décrit par une équation différentielle d'ordre supérieur à 3. Elle sera par contre réalisable par tout système dont l'équation différentielle est d'ordre inférieur ou égal à 3 . Pour un système d'ordre 4 ou 5 , il faudrait introduire le carré de la dérivée du jerk dans le critère. Au-delà, il faudrait introduire des dérivées d'ordre supérieur à 4.

\footnotetext{
${ }^{2}$ Remarque valable pour cette figure et les suivantes : l'écart de poursuite et ses oscillations viennent d'un écart de $20 \%$ entre la fréquence propre modélisée et la fréquence propre réelle. Cet écart est introduit volontairement pour mettre en évidence la faible sensibilité aux erreurs de modélisation.
}

La figure 2 représente le résultat de l'application de cette loi de mouvement à un système du $3^{\mathrm{e}}$ ordre, défini par l'équation différentielle (10) et la fonction de transfert (11) :

$$
\begin{gathered}
\frac{\tau}{\omega_{0}^{2}} \dddot{y}(t)+\left(\frac{1}{\omega_{0}^{2}}+\frac{2 \varsigma \tau}{\omega_{0}}\right) \ddot{y}(t)+\left(\frac{2 \varsigma}{\omega_{0}}+\tau\right) \dot{y}(t)+y(t)=u(t) \\
\frac{Y(\omega)}{U(\omega)}=\frac{1}{(1+\tau s)\left(1+2 \varsigma \frac{s}{\omega_{0}}+\frac{s^{2}}{\omega_{0}^{2}}\right)}
\end{gathered}
$$

Les paramètres utilisés pour calculer la loi de commande ont les valeurs suivantes : constante de temps $\tau=0,05 \mathrm{~s}$, pulsation propre $\omega_{0}=100 \mathrm{~s}^{-1}$ et coefficient d'amortissement $\zeta=0,01$. Le système simulé a la même constante de temps et le même amortissement, mais sa pulsation propre est égale à $120 \mathrm{~s}^{-1}$, ceci dans le but de tester la robustesse de la commande. L'amortissement est très faible, ce qui se traduit par une réponse indicielle fortement oscillatoire. L'application de la loi de commande 

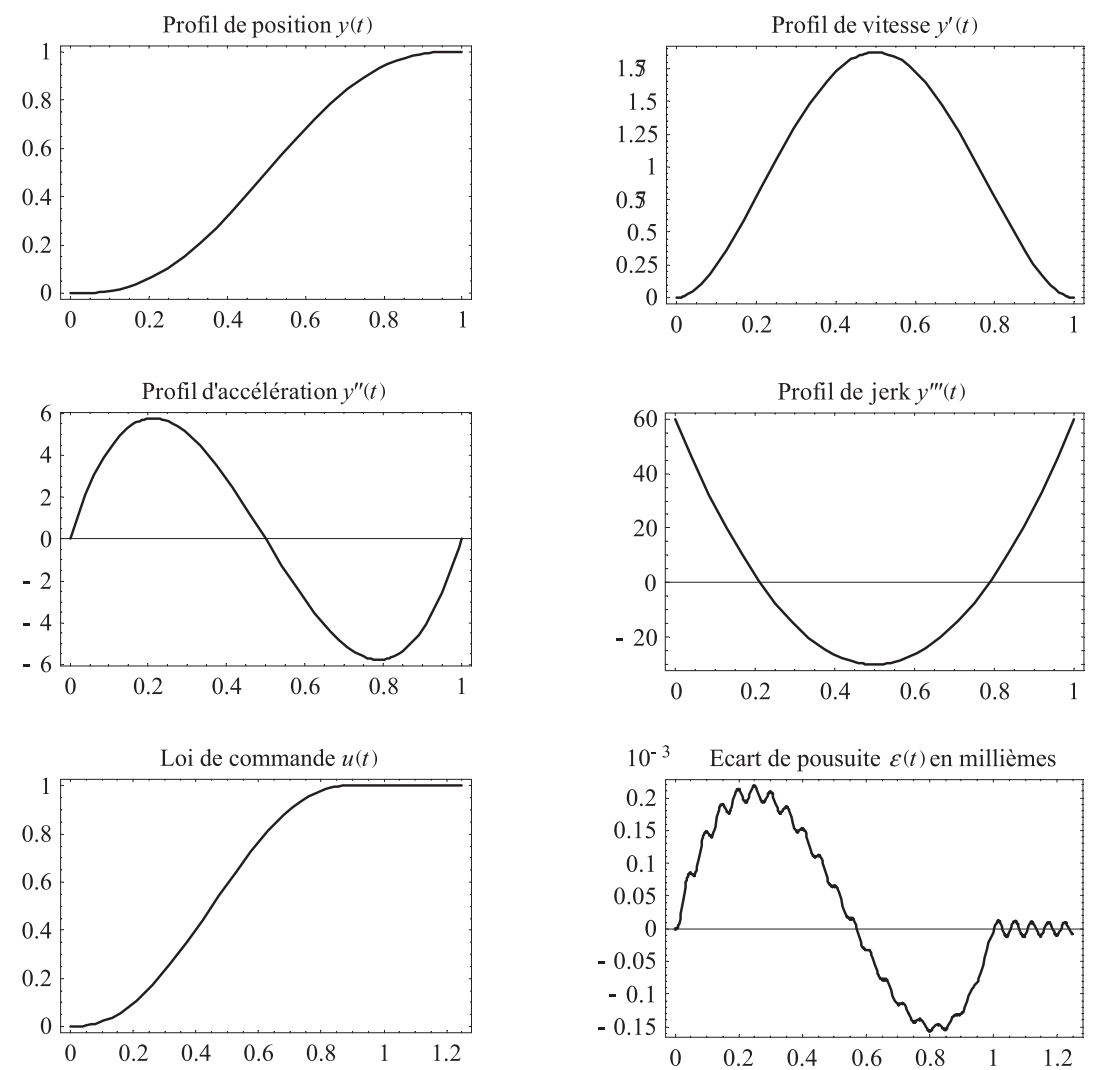

Fig. 3. Loi de mouvement à valeur efficace du jerk minimale et comportement du système simulé.

calculée à l'aide de l'équation (2) donne bien un mouvement dépourvu de toute oscillation lorsque les paramètres du système sont identiques à ceux de la loi de commande. Le fait qu'il y ait une différence de $20 \%$ sur la pulsation propre se traduit par de faibles oscillations qui ne sont pas visibles sur le tracé du mouvement simulé, mais seulement sur celui de l'écart de poursuite.

\subsection{Minimisation du jerk}

Lorsque l'on veut minimiser uniquement le jerk, et ceci au détriment de l'accélération et de la vitesse, il suffit de reprendre le critère (5) en annulant les coefficients de pénalisation de l'accélération et de la vitesse, soit : $\lambda_{\mathrm{a}}=$ 0 et $\lambda_{\mathrm{v}}=0$. Il n'est alors pas nécessaire de reprendre les calculs de l'optimisation, mais seulement l'équation finale (9) qui devient :

$$
::_{\mathrm{d}}(t)=0
$$

Ce résultat est très remarquable car l'intégration de cette équation différentielle donne un polynôme de degré 5 . Les six coefficients de ce polynôme se déterminent tout simplement par l'application des conditions de continuité de position, vitesse et accélération aux instants initiaux et finaux, ce qui donne six relations. La loi de mouvement ainsi obtenue présentera des discontinuités de jerk, tout comme la loi déterminée dans la section 2.2.
Dans les mêmes conditions que précédemment, mis à part le choix différent des coefficients de pénalisation, on obtient les lois de mouvement, de commande et d'écart de poursuite représentées figure 3 .

Par rapport au cas représenté figure 2, on constate que le jerk est beaucoup plus faible, que la vitesse maximale est sensiblement plus élevée et que l'accélération est à la fois plus faible et plus étalée dans le temps. La réduction du jerk se traduit par une réponse quasi exempte de vibration malgré la différence de $20 \%$ entre la pulsation propre utilisée pour calculer la loi de commande et celle du système simulé. Le fait que l'écart de poursuite soit sensiblement proportionnel à l'accélération s'explique par la discordance des pulsations propres. Cette discordance affecte de façon prépondérante le terme d'accélération de l'équation du mouvement (10) car l'amortissement et la constante de temps sont faibles.

\section{Lois de mouvement point à point par morceaux}

L'inconvénient de l'optimisation basée sur un critère énergétique ou pseudo-énergétique du type défini par l'équation (5) est de conduire à des lois de mouvement combinant des fonctions exponentielles et sinusoïdales ou à des lois de mouvement polynomiales d'ordre élevé. Ces types de lois de mouvement ne sont pas réalisables avec 

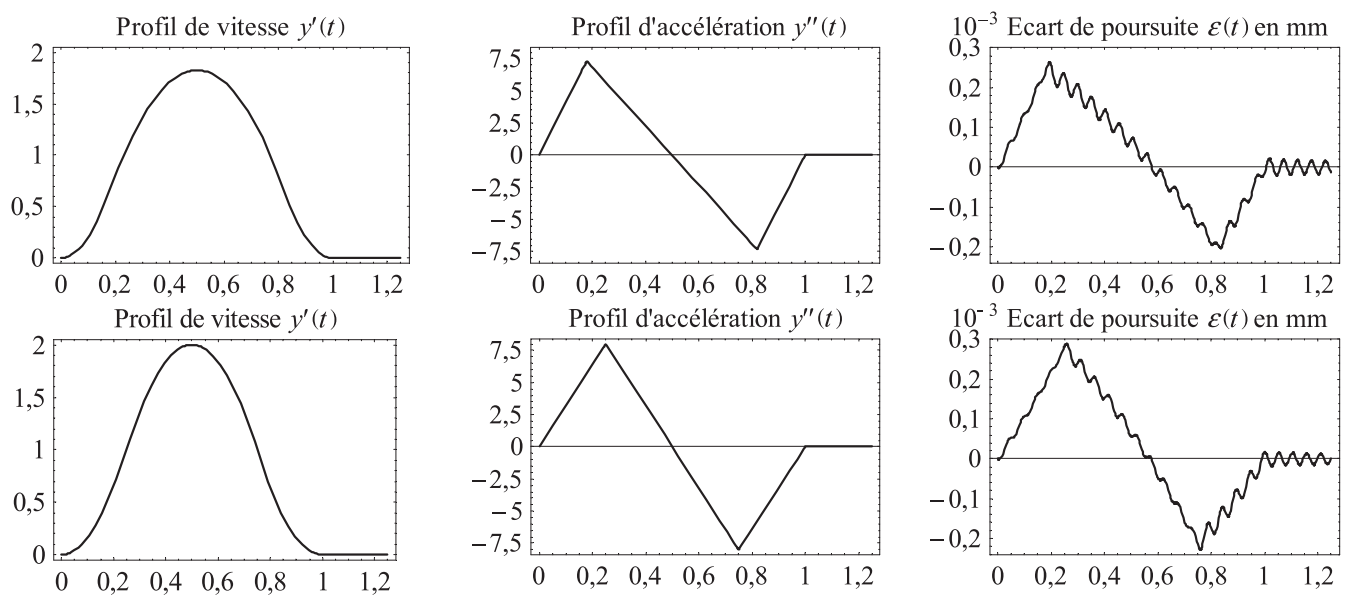

Fig. 4. Lois de mouvement cubiques et comportement du système simulé. Partie supérieure : loi minimisant l'intégrale du carré du jerk, partie inférieure : loi minimisant la valeur absolue du jerk.

les générateurs de trajectoire usuels dont sont dotés les directeurs de commande numérique.

L'utilisation d'un seul polynôme d'interpolation d'ordre $n$ permet de satisfaire $n+1$ conditions de continuité à répartir entre l'instant initial et final. Ainsi, la continuité de position, vitesse et accélération aux instants initiaux et finaux impose un polynôme de degré 5 . Mais, si on découpe l'intervalle de temps en 3 phases, successivement d'accélération, de conduite et de décélération et que l'on affecte un polynôme distinct à chacune de ces phases, il y a en tout 4 points de raccordement sur lesquels il faut assurer les 3 conditions de continuité, soit 12 conditions au total. La solution la plus simple est alors de choisir 3 polynômes du $3^{\mathrm{e}}$ degré, offrant chacun 4 paramètres ajustables. On obtient ainsi un système de 12 équations à 12 inconnues qui, sauf cas particulier, admet une solution unique.

La solution est une spline cubique. Ainsi, sur chacune des trois phases, le jerk est constant, l'accélération évolue linéairement et la vitesse paraboliquement. Cette spline ne peut pas être obtenue par les fonctions d'interpolation courantes, disponibles dans de nombreuses bibliothèques de fonctions mathématiques car celles-ci demandent que l'on donne les coordonnées des points et que l'on laisse libre les vitesses et accélérations. Mais la résolution du système d'équation permettant de déterminer la loi de mouvement est facile, car ces équations ont le bon goût d'être linéaires par rapport aux coefficients. Par contre, l'optimisation de la répartition du temps disponible entre les trois phases est délicate, la dépendance vis-à-vis du temps n'étant pas linéaire. Il serait trop long de développer les calculs ici, aussi nous contenterons-nous d'en donner le résultat dans le cas d'un mouvement unitaire effectué dans une durée unitaire. Pour un mouvement point à point, il n'y a aucun intérêt à choisir une loi de mouvement dissymétrique qui brutaliserait plus le système au début ou à la fin du mouvement. On notera $t_{\mathrm{a}}$ la durée de chacune des phases d'accélération et de décélération. La durée de la phase de conduite sera alors égale à $1-2 t_{\mathrm{a}}$. Les valeurs successives du jerk sont alors :

$$
\left\{\begin{array}{lll}
\frac{6}{t_{\mathrm{a}}\left(1-t_{\mathrm{a}}\right)} & \frac{-12}{2 t_{\mathrm{a}}^{2}-3 t_{\mathrm{a}}+1} \quad \frac{6}{t_{\mathrm{a}}\left(1-t_{\mathrm{a}}\right)}
\end{array}\right\}
$$

Il reste à choisir la durée des phases d'accélération et décélération. Un choix intéressant est celui qui minimise le jerk. On a le choix entre :

- la minimisation de l'intégrale du carré du jerk, ce qui donne

$t_{\mathrm{a}}=0,18$ et jerk $=\{40,68,-22,85,40,68\}$

- la minimisation de la valeur absolue du jerk, ce qui donne

$t_{\mathrm{a}}=0,25$ et jerk $=\{32,-32,32\}$

La figure 4 permet de comparer les performances des deux critères. L'écart de poursuite est calculé pour un système simulé ayant une pulsation propre de $120 \mathrm{~s}^{-1}$, soit $20 \%$ d'écart par rapport à la pulsation propre utilisée pour le calcul de la loi de commande.

On vérifie que le critère qui minimise la valeur absolue du jerk provoque moins de vibration. Par contre, il provoque plus d'écart de poursuite, ce qui s'explique par le fait que l'accélération variant plus lentement au début et à la fin du mouvement, il faut atteindre une accélération et une vitesse plus élevées au cours du mouvement pour réaliser celui-ci dans le même laps de temps.

En rapprochant ces résultats de ceux présentés figure 3, on constate que bien qu'étant plus simple et « sousoptimale »du fait que l'on se prive de la possibilité de faire varier le jerk progressivement, la loi de mouvement de type spline cubique donne des résultats presque aussi performants pour l'écart de poursuite et équivalents pour la minimisation des vibrations.

\section{Conclusions et perspectives}

Les lois de mouvement présentées dans cet article sont toutes déterminées par optimisation à partir de divers 
critères. L'optimisation est une démarche plus rationnelle que le choix a priori d'une méthode d'interpolation et l'affectation intuitive de valeurs à ses paramètres.

Les résultats présentent plusieurs caractéristiques intéressantes lorsqu'on cherche à réduire les écarts de poursuite et les vibrations :

1. Mis à part pour les systèmes à déphasage non minimal qui nécessitent une étude plus approfondie, il est établi que n'importe quelle loi de mouvement convient dès lors qu'elle est dérivable, y compris aux instants initiaux et finaux, avec des dérivées pas trop grandes jusqu'à un degré égal à l'ordre de la fonction de transfert.

2. Le critère d'optimisation peut être choisi indépendamment des paramètres de la fonction de transfert. L'intérêt majeur est que la loi de mouvement qui résulte de l'optimisation est indépendante des caractéristiques spécifiques à l'axe asservi, mis à part l'ordre de sa fonction de transfert. Seule la loi de commande dépend des paramètres de la fonction de transfert, mais le réglage de ses paramètres de calcul n'est pas critique.

3. Les lois polynomiales respectant les conditions de continuité jusqu'au degré $n-1$ de dérivation sont applicables à tout système d'ordre inférieur ou égal à $n$. Ainsi, un générateur de mouvement d'ordre $n$ est «n-universel », au sens qu'il est apte à conduire sans engendrer de vibration tous les systèmes ayant une fonction de transfert d'ordre inférieur ou égal à $n$.

L'interpolation par spline cubique, qui a l'avantage d'être incluse dans les systèmes de commande numérique actuels, permet ainsi de piloter sans générer d'écart de poursuite ni de vibration des axes asservis dont la fonction de transfert est d'ordre 3 au maximum. Au-delà, il faut s'attendre à avoir des écarts et des vibrations. Pour un axe asservi présentant deux modes vibratoires, si on ne veut exciter aucun des deux modes, il faut passer à des splines d'ordre 5 ... Mais, si on n'augmente pas l'ordre de l'interpolateur, du fait que la loi de mouvement générée est la plus « douce » possible, les modes négligés lors de l'établissement des lois mouvement et de commande restent peu sollicités.

En perspectives de l'étude présentée ici, il reste donc à développer une approche permettant d'évaluer l'amplitude de l'écart de poursuite des vibrations causées par l'approximation du modèle, à la fois du point de vue des modes négligés et des incertitudes sur les paramètres du système.

Une autre voie d'investigation est la génération de trajectoire dans le plan ou dans l'espace. Elle ne peut plus se faire par simple raccordement de segments de droite et d'arcs de cercles qui induisent des changements de courbure et donc des discontinuités d'accélération d'autant plus fortes que la vitesse est élevée. Une idée serait d'échantillonner la trajectoire spécifiée et d'interpoler par une spline cubique les points ainsi obtenus. La trajectoire ainsi reconstruite ne serait certes pas identique à la spécification initiale, mais elle ne s'en écarterait que très peu. Et, comme elle serait suivie sans écart de poursuite ni à-coup générateur de vibrations, la qualité du résultat aurait toutes les chances d'être nettement améliorée, probablement plus proche de la spécification qu'en voulant violer les incontournables lois de la mécanique. Une autre idée serait d'inclure les contraintes de la génération de mouvement le plus en amont possible, dans le processus de conception, en même temps que la génération de trajectoire telle que le préconisent Emmanuel Duc et al. dans [8].

\section{Références}

[1] A. Dequidt, M. Gauthier, E. Valdes, W. Khalil, Intégration de la loi de mouvement $\mathrm{CN}$ lors de la conception mécanique d'une machine cartésienne. Assises Machines et Usinage à Grande Vitesse, Association pour le travail à grande vitesse, CETIM Senlis, 6, 7 mars 2000

[2] F. Debrouke, Contribution à la maîtrise du basculement dans les machines cartésiennes; application aux machines dédiées à l'usinage à grande vitesse, Thèse de Doctorat, Université de Valenciennes, novembre 2003

[3] D. Brun-Picard, F. Gérardin, Évolution des techniques de commande d'axes pour améliorer les performances en usinage grande vitesse, Assises Machines et Usinage à Grande Vitesse, CETIM Senlis, 6, 7 mars 2000

[4] D. Brun-Picard, F. Malburet, Contrôle actif des vibrations, ce qui marche et ce qui ne marche pas, $2^{\text {es }}$ Assises Machines et Usinage à Grande Vitesse, ENSAM Lille, 13, 14 mars 2002

[5] S. Bloch, Gestion des vitesses par la commande numérique en usinage grande vitesse, Assises Machines et Usinage à Grande Vitesse, CETIM Senlis, 6, 7 mars 2000

[6] S. Bloch, E. Deneuville, L. Tan, Optimisation de la vitesse d'avance en commande numérique, $2^{\text {es }}$ Assises Machines et Usinage à Grande Vitesse, ENSAM Lille, 13, 14 mars 2002

[7] R. Béarée, P.J. Barre, Influence du jerk sur le comportement dynamique d'un robot cartésien 3 axes, $3^{\text {es }}$ Assises Machines et Usinage à Grande Vitesse, IFMA ClermontFerrand, 10, 11 mars 2004, Mécanique \& Industries 6 (2005) 439

[8] E. Duc, C. Lartigue, P. Bourdet, Utilisation de l'interpolation polynômiale pour l'UGV. Cas de l'usinage à 5 axes, $2^{\text {es }}$ Assises Machines et Usinage à Grande Vitesse, ENSAM Lille, 13, 14 mars 2002 